\title{
Multispectral Optoacoustic Tomography of Matrix Metalloproteinase Activity in Vulnerable Human Carotid Plaques
}

\author{
Daniel Razansky, ${ }^{1}$ Niels J. Harlaar, ${ }^{1,2}$ Jan Luuk Hillebrands, ${ }^{3}$ Adrian Taruttis, ${ }^{1}$ \\ Eva Herzog, ${ }^{1}$ Clark J. Zeebregts, ${ }^{2}$ Gooitzen M. van Dam, ${ }^{2}$ Vasilis Ntziachristos ${ }^{1}$ \\ ${ }^{1}$ Institute for Biological and Medical Imaging, Technische Universität München and Helmholtz Zentrum München, Munich, Germany \\ ${ }^{2}$ Department of Surgery and Bio-optical Imaging Center Groningen, University Medical Center Groningen, Groningen, The Netherlands \\ ${ }^{3}$ Department of Pathology and Medical Biology, Pathology Division, University Medical Center Groningen, Groningen, The Netherlands
}

\begin{abstract}
Aims: Elevated expression of cathepsins, integrins and matrix metalloproteinases (MMPs) is typically associated with atherosclerotic plaque instability. While fluorescent tagging of such molecules has been amply demonstrated, no imaging method was so far shown capable of resolving these inflammation-associated tags with high fidelity and resolution beyond microscopic depths. This study is aimed at demonstrating a new method with high potential for noninvasive clinical cardiovascular diagnostics of vulnerable plaques using high-resolution deep-tissue multispectral optoacoustic tomography (MSOT) technology.

Methods and results: MMP-sensitive activatable fluorescent probe (MMPSense ${ }^{\mathrm{TM}}$ 680) was applied to human carotid plaques from symptomatic patients. Atherosclerotic activity was detected by tuning MSOT wavelengths to activation-dependent absorption changes of the molecules, structurally modified in the presence of enzymes. MSOT analysis simultaneously provided morphology along with heterogeneous MMP activity with better than 200 micron resolution throughout the intact plaque tissue. The results corresponded well with epifluorescence images made from thin cryosections. Elevated MMP activity was further confirmed by in situ zymography, accompanied by increased macrophage influx.

Conclusions: We demonstrated, for the first time to our knowledge, the ability of MSOT to provide volumetric images of activatable molecular probe distribution deep within optically diffuse tissues. High-resolution mapping of MMP activity was achieved deep in the vulnerable plaque of intact human carotid specimens. This performance directly relates to pre-clinical screening applications in animal models and to clinical decision potential as it might eventually allow for highly specific visualization and staging of plaque vulnerability thus impacting therapeutic clinical decision making.
\end{abstract}

Key words: Atherosclerosis, Optoacoustic imaging, Carotid arteries, Plaque, Contrast media, Inflammation

Abbreviations: CEA carotid endarterectomy; DQ dye-quenched; MSOT multispectral optoacoustic tomography; MMP matrix metalloproteinases; OCT optical coherence tomography; CCD cooled charge-coupled device

N.J. Harlaar and J.L. Hillebrands contributed equally to this work.

Correspondence to: Daniel Razansky; e-mail: dr@tum.de, Clark Zeebregts; e-mail: czeebregts@hotmail.com

\section{Introduction}

A therosclerotic plaque progression is clearly correlated to increased levels of inflammation [1]. In particular, 
the appearance of matrix metalloproteinases (MMPs), and especially of MMP-9, in carotid plaque is associated with high risk vulnerability [2-4]. Even though plaque anatomy and physiology can be visualized by different methods such as duplex ultrasound [5], computed tomography scan [6], magnetic resonance imaging [7] or intravascular optical coherence tomography (OCT) [8], imaging of core biological processes involved in plaque formation on a cellular and molecular level, e.g., MMP and protease up-regulation, is limited with these modalities.

Detection of MMP activity in plaques is possible using the regular planar fluorescence imaging techniques, assisted with smart activatable molecular probes, e.g.,

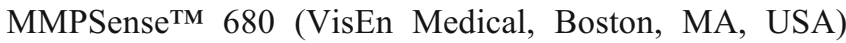
[9]. However, due to intense light scattering in tissues as well as a complicated morphology of common plaques, the planar fluorescence methods, currently available for optical imaging, can at best provide a qualitative indication of the existence of MMP activity rather than an accurate assessment of its specific location (i.e., threedimensional morphology) and distribution. However, if fluorescence imaging is to be implemented as a diagnostic clinical tool, it would require ability to three-dimensionally image and accurately quantify not only deep seated plaque anatomy measures but also its physiological and molecular constitution through several millimeters to centimeters of tissue.

In the recent study by Levi et al. [10], detection of optoacoustic signal from a cell culture incubated with activatable optoacoustic probe, sensitive to enzymatic cleavage by MMP-2, has been reported. Here, we considered whether multispectral optoacoustic tomography (MSOT) [11] could be employed for imaging of atherosclerotic biomarkers in human carotid arteries by resolving the activation of previously documented smart probe molecules specific to enzymes involved in atherosclerosis [12]. Such ability could then allow the detection of such probes with unprecedented imaging quality compared to conventional fluorescence imaging techniques. This is because MSOT has been recently shown to accurately resolve both intrinsic tissue morphology along with extrinsically administered molecular agents [11] and fluorescent proteins [13] deep in highly scattering biological tissues with spatial resolution down to around $40 \mu \mathrm{m}$, a metric characteristic of diffraction limited ultrasonic imaging [14]. The technique illuminates the imaged object using pulsed light at multiple wavelengths and records the ultrasonic signals generated in response to light absorption in a tomographic detection setting [11]. Tomographic reconstruction and spectral processing allow then three-dimensional imaging of tissue chromophores and extrinsic bio-markers with distinct spectral signatures [15]. In this work we demonstrate that by utilizing the spectral ability of the technique, absorption changes indicative of conformational changes of the probe molecules utilized, due to interaction of the probe molecule with underlying enzymes present in disease, can be detected and subsequently threedimensionally reconstructed with high accuracy.

\section{Methods}

\section{Endarterectomy Specimens and Fluorescence Labeling}

Standard surgical carotid endarterectomy (CEA) was performed on five patients with a symptomatic $>70 \%$ carotid stenosis at the University Medical Center Groningen. All investigations conformed with the principles outlined in the Declaration of Helsinki. The freshly isolated CEA specimens were acquired with approval of the local Ethical Committee and informed consent of the patients. Immediately after endarterectomy, the plaques were incubated for $1 \mathrm{~h}$ in a $10-\mu \mathrm{M}$ concentration suspension of MMPsensitive activatable probe (MMPSense ${ }^{\mathrm{TM}}$ 680; VisEn Medical, Boston, MA, USA) at $37^{\circ} \mathrm{C}$. The control plaques were instead incubated in PBS for $1 \mathrm{~h}$ at $37^{\circ} \mathrm{C}$. MMPSense $\mathrm{MM}^{\mathrm{TM}} 680$ is a probe belonging in a class of activatable 'smart' probes that is dark (minimally fluorescent in its base state) but exhibits fluorescence properties after conformation changes occurring in the presence of different enzymes, preferentially MMP2/9 and trypsin and to a lesser extent of other proteases. Furthermore, once activated, MMPSense is a potential candidate for optoacoustic imaging since, similarly to most other far-red and near-infrared fluorescent agents and dyes; it has a relatively low quantum yield and therefore more than $80 \%$ of its extinction energy is transferred into optoacoustic signals. After endarectomy and incubation with MMPSense the plaque was snap frozen in liquid nitrogen allowing shipment. Subsequently, it was defrosted and analyzed by Rotational Planar Fluorescence Imaging and MSOT after which the specimen was snap frozen again in liquid nitrogen for further immunohistochemical analysis.

\section{Rotational Planar Fluorescence Imaging}

To confirm the presence of activation in the carotid samples considered, specimens were scanned on a rotational planar fluorescence imaging system, just before the MSOT imaging. The samples were placed vertically on a stepper-motor-driven rotation stage, and were illuminated at $675 \mathrm{~nm}$. Fluorescence images were collected at multiple angles, after sample rotation in front of the camera, using an ultra-low noise cooled chargecoupled device (CCD) camera (VersArray, Roper Scientific, Trenton, NJ, USA) with a $512 \times 512$ pixel array, through a bandpass fluorescence $(710 \pm 5 \mathrm{~nm}$; Andover., Salem, NH, USA). The samples were rotated by $3^{\circ}$ steps in the range of $0-360^{\circ}$, each using an exposure time of $5 \mathrm{~s}$.

\section{Phantom Preparation}

Experimental demonstration of the ability to resolve activated probes by MSOT in a controlled experiment, we prepared and imaged a phantom moulded from $1.3 \%$ of agar powder (SigmaAldrich, St. Louis, MO, USA) into a cylinder of 14-mm diameter. Tissue-like scattering properties were introduced by mixing $12 \%$ of 
Intralipid 20\% emulsion (Sigma-Aldrich) in the agar. Two cylindrical openings with diameters of approximately $3 \mathrm{~mm}$ were introduced directly into the phantom, both openings containing MMPSense at $1 \mu \mathrm{M}$ concentration. The probe in the right opening was activated using $100 \mu \mathrm{M}$ Trypsin without phenol red indicator (Invitrogen, Germany), whereas the probe in the left opening was incubated with PBS solution only and was in its inactive state. After $4 \mathrm{~h}$ incubation at room temperature, the absorption spectrum of both the activated and inactive control solution was measured using a VIS-NIR spectrometer (USB 2000; Ocean Optics, Dunedin, FL, USA). A planar fluorescence image of the solutions was recorded to verify probe activation.

\section{Optoacoustic Signal Acquisition}

Multiwavelength MSOT experimental setup, shown in Fig. 1, was based on a tunable MOPO laser (Quanta-Ray MOPO-700; SpectraPhysics, Mountain View, CA, USA) pumped by a Q-switched Nd: YAG laser (Quanta-Ray Lab-Series 190-30; Spectra-Physics) operating at its third harmonic $(355 \mathrm{~nm})$. The pulse duration of the laser was less than $10 \mathrm{~ns}$ and the repetition rate is $30 \mathrm{~Hz}$. The output laser beam size was adjusted to fit the size of the specimen. The 50/50 beam splitter (BSW16; Thorlabs) splits the beam into two equal-intensity parts being guided from two opposite directions onto the object's surface through two transparent windows in the imaging tank filled with water. In this way, optimal excitation conditions close to uniform illumination were achieved. The estimated light fluence upon the surface of the specimen was $2 \mathrm{~mJ} / \mathrm{cm}^{2}$, a level well below the permissible exposure limit [16] of $20 \mathrm{~mJ} / \mathrm{cm}^{2}$ at the wavelength range used here for the imaging. A

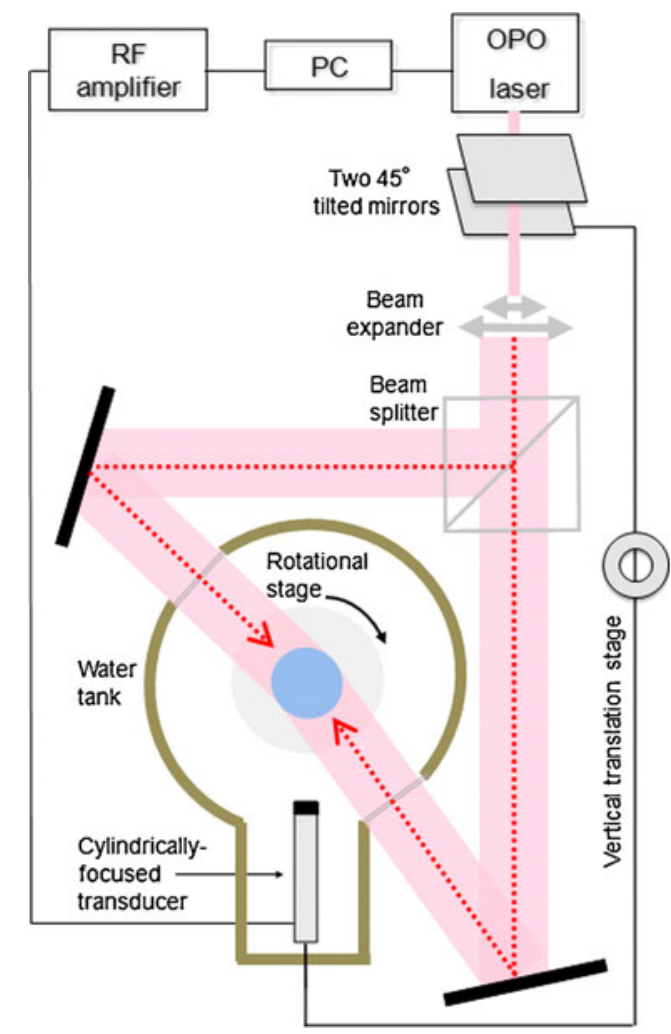

Fig. 1. Schematics of the multispectral optoacoustic tomography (MSOT) experimental setup. wideband piezoelectric PZT transducer (V319, 3.5 MHz central frequency, Panametrics-NDT UT Transducers, Olympus) was used to detect optoacoustic signals from the illuminated sample. The transducer was cylindrically focused in the imaging plane $(38 \mathrm{~mm}$ focal distance) to allow 3D data acquisition via vertical scanning. Two $45^{\circ}$ tilted mirrors were used to change the beam height by moving the bottom mirror with a vertical translation stage. The beam splitter and the ultrasonic transducer were translated by the same stage, thus both the illumination and detection planes were translated simultaneously to allow for 3D image acquisition via vertical scanning. The samples were mounted on a rotational stage so that in-plane tomographic data acquisition was done by $360^{\circ}$ rotation of the sample. A 14-bit resolution PCI digitizer with a sampling rate of $100 \mathrm{MS} / \mathrm{s}$ (NI PCI-5122; National Instruments, Austin, TX, USA) was used to record the time-resolved acoustic signals detected by the transducer. We placed a photodiode (FDS010, 200-1100 nm, 1 ns Rise Time; Thorlabs) in the vicinity of the laser output window and recorded the intensity change of scattered light for each pulse in order to normalize the detected signals for laser output instabilities. In this way, quantitative readings of pulse-to-pulse laser intensity variations over broad wavelength range were obtained by calibrating the measurements using spectral response curve of the diode. This continuous power monitoring was of critical importance for multispectral reconstructions since some of important biomarkers may present only a small variation of the optical absorption over highly absorbing background, in which case even small quantification inaccuracies may lead to flawed results. The laser, stage controllers, and data acquisition were all synchronized via Labview-based interface (National Instruments). Single-wavelength two-dimensional data acquisition took approximately 30 s. Generation of a full $3 \mathrm{D}$ data set with, e.g., three wavelengths and ten vertical slices would take about $20 \mathrm{~min}$. Image reconstruction for each vertical slice required $3 \mathrm{~s}$ and was performed by using a two-dimensional filtered back-projection (cylindrical Radon) algorithm [17].

\section{Multispectral Optoacoustic Tomography and Detection of Enzymatic Activation}

MSOT can distinguish the activated probe, over both slowly varying spectra of the background and the inactive probe, by acquiring optoacoustic data at several wavelengths and analyzing spectral contributions on a per-pixel bases, i.e.,

$$
\mu_{\mathrm{a}}^{j}(\lambda)=\varepsilon_{\mathrm{a}}(\lambda) c_{\mathrm{a}}^{j}+\varepsilon_{\mathrm{i}}(\lambda) c_{\mathrm{i}}^{j}+B(\lambda)
$$

where $\mu_{\mathrm{a}}^{j}(\lambda)$ is wavelength-dependent absorption in pixel $j$ as reconstructed by optoacoustic tomography, $B(\lambda)$ is contribution of background chromophores, $\varepsilon_{\mathrm{a}}(\lambda), \varepsilon_{i}(\lambda), c_{\mathrm{a}}^{j}$, and $c_{\mathrm{i}}^{j}$ are extinction of activated and inactive probe and their spatial distribution on a per pixel basis. Probe distribution (both activated and inactive) can subsequently be found by, e.g., applying linear regression to Eq. 1 at multiple wavelengths. However, in the particular case studied here, we observed that inactive MMPSense exhibits similar absorption (extinction) at 635 and 675 nm, i.e., $\varepsilon_{i}(675) \approx \varepsilon_{i}(635)$. By further assuming slow 
background variations, Eq. 1 is readily reduced into an explicit expression for the activated probe distribution

$$
c_{\mathrm{a}}^{j}=\frac{\mu_{\mathrm{a}}^{j}(675)-\mu_{\mathrm{a}}^{j}(635)}{\varepsilon_{\mathrm{a}}(675)-\varepsilon_{\mathrm{a}}(635)}=\frac{\Delta \mu}{\Delta \varepsilon}
$$

leading to an effective suppression of the contribution from the inactive probe. Distribution of the latter can also be resolved using a similar procedure. Nevertheless, it is presumed that in more complicated cases, especially in the presence of high background variations of whole blood in living tissues, true multispectral fitting procedures (using full version of Eq. 1 including known spectra of blood) might become necessary for effective background suppression.

\section{Cryosectioning and Epi-Fluorescence Imaging}

Immediately following the MSOT and fluorescence imaging session, the agar-embedded specimen was placed into a $30 \mathrm{~mm}$ $\varnothing$ syringe (Falcon, Becton Dickinson, Franklin Lakes, NJ, USA) and freezing medium was added. The syringe and its content were snap-frozen at $-80^{\circ} \mathrm{C}$. After freezing, cryosections through the entire sample were made with $50-\mu \mathrm{m}$ intervals. For every $1 \mathrm{~mm}$, the thickness of the sections was reduced to $10 \mu \mathrm{m}$ providing sections suitable for additional histological analysis. Selective $50-\mu \mathrm{m}$ sections (approx. every $1 \mathrm{~mm}$ ) were imaged by an epi-fluorescence method to attain scattering-free images of fluorescence activity in each thin section.

\section{In Situ Zymography}

In order to confirm presence of active MMPs in areas with increased fluorescence, as identified by MSOT, a cryosection $(10 \mu \mathrm{m})$ from the corresponding area was subjected to in situ zymography to visualize gelatinase activity. To this end, the section was incubated with $\mathrm{DQ}^{\mathrm{TM}}$ gelatin $\left(100 \mu \mathrm{g} / \mathrm{mL}\right.$, EnzCheck ${ }^{\circledR}$ Gelatinase/Collagenase Assay Kit; Invitrogen-Molecular Probes, Breda, the Netherlands) dissolved in $1 \%$ agarose (in PBS, Type IX, Ultra-low Gelling Temperature, Sigma-Aldrich Chemie, Zwijndrecht, the Netherlands) overnight at $37^{\circ} \mathrm{C} .4^{\prime}, 6$-Diamidino-2-phenylindole dihydrochloride (DAPI) was added to the $1 \%$ agarose solution to stain nuclei. To control for autofluorescence, a serial section was incubated with $1 \%$ agarose/PBS/DAPI in the absence of $\mathrm{DQ}^{\mathrm{TM}}$ gelatin. Sections were analyzed on a Leica DMLB fluorescence microscope (Leica Microsystems, Rijswijk, the Netherlands) equipped with a Leica DC300F camera and LeicaQWin 2.8 software.

\section{Immunohistochemistry for CD68}

Cryosections $(10 \mu \mathrm{m})$ from areas with differential MMP activity as identified by MSOT and in situ zymography were analyzed for the presence of infiltrated $\mathrm{CD}^{+} 8^{+}$macrophages using immunohistochemistry. Frozen sections were thawed, air-dried and then acetonefixed for $10 \mathrm{~min}$ at room temperature. After fixation, sections were air-dried followed by endogenous peroxidase blockade $\left(0.3 \% \mathrm{H}_{2} \mathrm{O}_{2}\right.$ in PBS, 15 min room temperature). Next, sections were rinsed in PBS and blocked in $10 \%$ rabbit serum $/ 1 \%$ BSA/PBS for $15 \mathrm{~min}$.
After blocking endogenous avidin/biotin (Avidin/Biotin blocking kit; Vector Laboratories, Burlingame, CA, USA) the sections were incubated with mouse-anti-human CD68 (clone KP1; DAKO Netherlands, Heverlee, Belgium) in $1 \%$ BSA/PBS for $60 \mathrm{~min}$, room temperature. Following primary antibody incubation, sections were rinsed in PBS $(3 \times 5 \mathrm{~min})$ after which they were incubated with biotin-conjugated rabbit-anti-mouse polyclonal antibody (DAKO) in $1 \% \mathrm{BSA} / \mathrm{PBS}$ for $30 \mathrm{~min}$, room temperature. After rinsing in PBS $(3 \times 5 \mathrm{~min})$ sections were incubated with HRP-conjugated streptavidin (DAKO) for $30 \mathrm{~min}$, room temperature. Peroxidase activity was visualized with 3-amino-9-ethylcarbazole (AEC). Nuclei were counterstained with hematoxylin and sections were embedded in Kaiser's glycerin-gelatin. Sections were analyzed on an Olympus BX50 microscope (Olympus Nederland, Zoeterwoude, the Netherlands) equipped with an Olympus DP70 camera and Cell B imaging software.

\section{Results}

\section{Phantom Validation Studies}

Figure 2 demonstrates basic validation measurements that summarize and explain the MSOT ability to detect probe enzymatic activation. Fig. 2a plots measurements of the spectral extinction characteristics of the inactive (dotted line) and activated (solid gray line) forms of the MMPSense ${ }^{\mathrm{TM}}$ 680 probe while the spectra of oxy- and deoxyhemoglobin is also shown for reference purposes. An enabling feature for the method developed herein, is the characteristic spectral change of the extinction (absorption) coefficient between activated and non-activated states, in the far-infrared portion of the spectrum between 635 and $675 \mathrm{~nm}$, shown in greater detail in the insert of Fig. 2a.

To test the methodology, the tissue-mimicking (scattering and absorbing) phantom with insertions containing activated and inactive MMPSense probe was imaged by both MSOT and epi-fluorescence. While spectral changes between active (circular insertion on the right) and inactive (insertion on the left) are not clearly distinguishable at the representative single wavelength reconstructions of Fig. $2 b$ (made at $635 \mathrm{~nm}$ ) and Fig. 2c $(675 \mathrm{~nm})$ of the phantom imaged, multispectral processing accurately reveals the different states between the left and right tubes in Fig. 2d. Fig. 2e shows a corresponding 'top-view' planar fluorescence image of the phantom, made after the MSOT measurements, showing strong fluorescence from the insertion containing the activated probe. Interestingly, light diffusion through the phantom causes the appearance of background diffusive fluorescence signal on Fig. 2e, which is absent from the reconstructed MSOT images of Fig. $2 d$ due to the high-resolution three-dimensional imaging capabilities of the method, not affected by light diffusion. Finally, Fig. 2f offers an independent confirmation of the MMPSense activation by trypsin and the fluorescence signal changes observed by direct imaging of activation in two transparent tubes, without surrounding diffusive medium. 

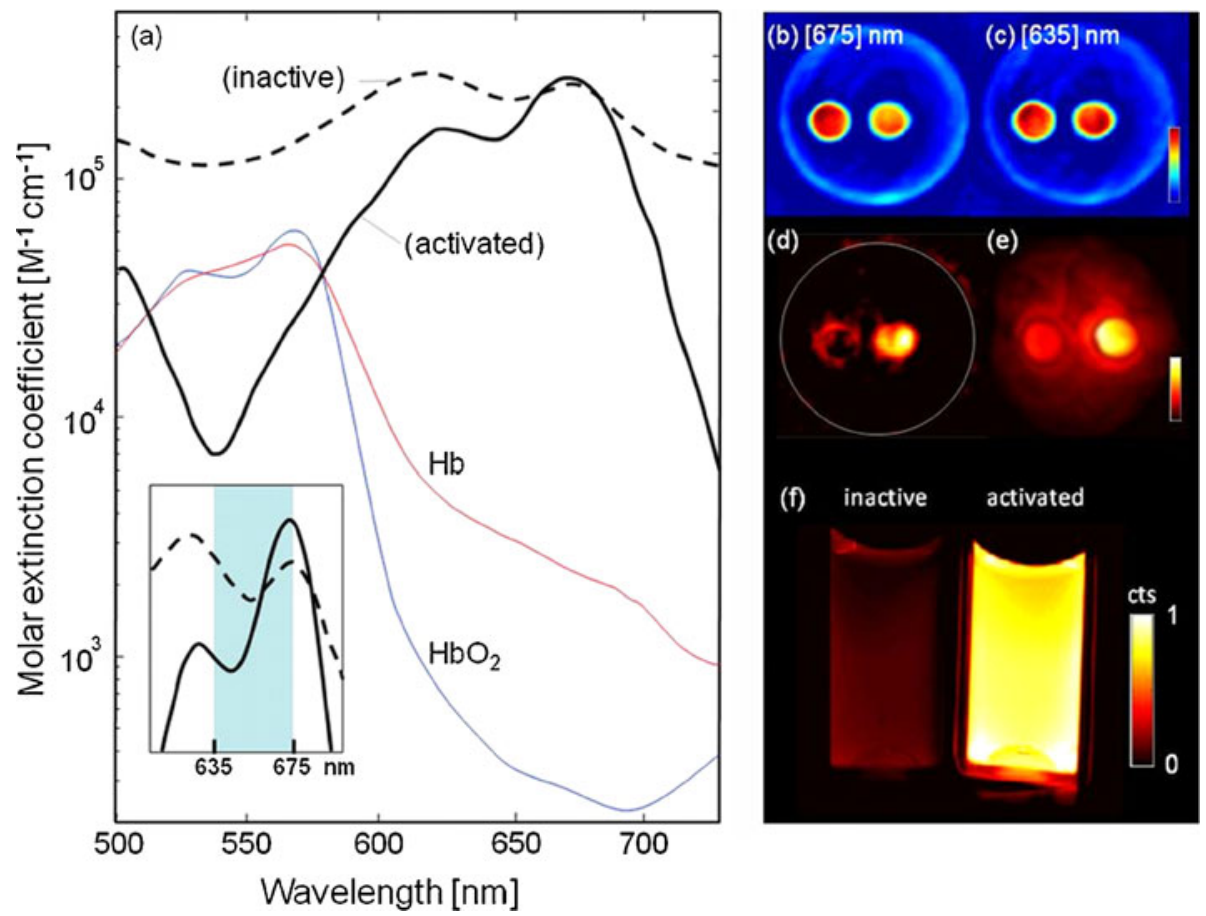

Fig. 2. a Measured spectral extinction characteristics of inactive (dotted line) versus activated (solid gray line) MMPSense ${ }^{\mathrm{TM}}$ 680 probe. Spectra of oxy- and deoxyhemoglobin are also shown for reference. Inset: zoom-in from the wavelength range that was used for MSOT imaging. b Optoacoustic image of phantom with MMPSense ${ }^{\mathrm{TM}}$ insertions at $635 \mathrm{~nm}$ excitation. Circular insertion containing activated probe is on the right while the inactive insertion is on the left. c Corresponding optoacoustic image at $675 \mathrm{~nm}$ excitation. d Multispectrally resolved (MSOT) image resolving the distribution of activated probe inside the phantom. e Planar (top view) fluorescence image of the phantom performed after MSOT imaging sessions, confirming fluorescence from the activated probe. $f$ Planar fluorescence image comparing inactive (left) and active MMPSense ${ }^{\mathrm{TM}}$.

\section{Imaging of Plaque Morphology and Planar Fluorescence}

Figure $3 b-d$ presents rotational images taken from a representative intact plaque sample (shown in Fig. 3a), from different view angles. Normally, MMP (fluorescence) activity should be mostly concentrated around the carotid artery bifurcation area, where most of the vulnerability is expected [18]. However, common plaques represent geometrically complex and highly heterogeneous structures, having multiple openings and large variations in vessel wall thickness and optical properties of different components. The multiple projection images and the rotational movie (available as Supplementary Information online) evince that the excitation and emission light have been guided and attenuated non-uniformly in various areas of the plaque. This readily leads to erroneous conclusions regarding the origin of the fluorescent signals that are being detected by the CCD camera located outside the sample and in any case does not allow for a three-dimensional understanding of the disease extent. As an example, some of the light in Fig. $3 \mathrm{~d}$, that is thought as coming from the opening of the external carotid (branch) artery, could in fact originate from another location deep inside the plaque and guided through scattering-free areas. In another projection, shown in Fig. 3c, the bifurcation area does not show high levels of fluorescence. The likely explanation for this observation is that the branch artery is located relatively far away from the surface that is facing the CCD camera resulting in high attenuation of the light as it travels through a highly scattering and absorbing plaque area. The inaccuracy of planar fluorescence imaging has been previously discussed [19].

Figure $3 \mathrm{e}$ shows three-dimensional morphological optoacoustic images resulting from an intact plaque. These data are obtained at a single wavelength of $635 \mathrm{~nm}$ at which activated MMPSense has low excitation efficiency so that mostly morphological information but not MMP activity can be extracted from the images. The morphologic characteristics have in-plane resolution on the order of $200 \mu \mathrm{m}$, limited by the useful bandwidth of the ultrasonic detector. Images at four different levels around the bifurcation area, all separated by $2 \mathrm{~mm}$, are shown, revealing good correspondence to the color photographs of a sliced plaque (Fig. 3f). The 50- $\mu \mathrm{m}$ cryosections were obtained approximately from the same area where optoacoustic images were produced.

\section{Three-Dimensional Visualization of MMP Activity from an Intact Specimen with MSOT}

In order to resolve MMP activity with high-resolution over non-specific intrinsic tissue background and inactive probe 

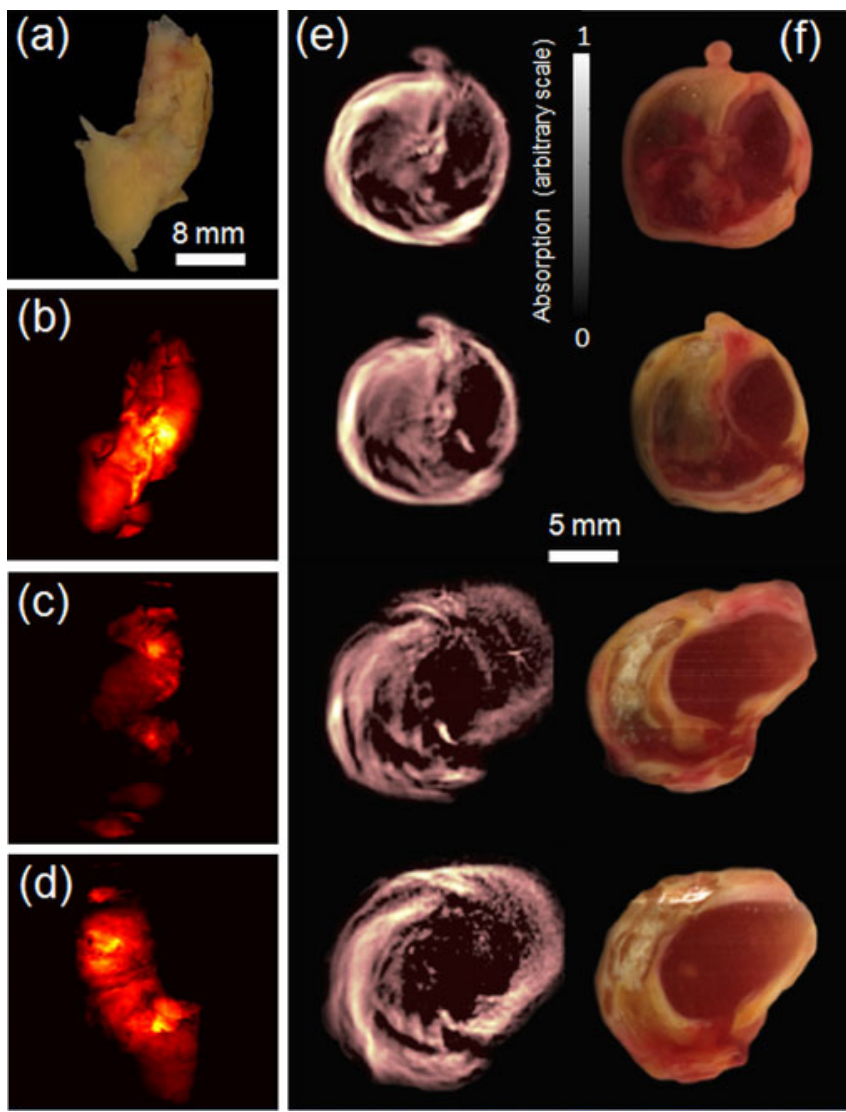

Fig. 3. Rotational planar fluorescence imaging of an intact specimen and plaque morphology. a Color image of a human carotid specimen. b-d Fluorescence images from the same specimen embedded in clear agar phantom rotated with $90^{\circ}$ steps. See also Video online of continuously rotated specimen. e Three-dimensional stack (2-mm vertical steps) of morphological optoacoustic images made from an intact specimen using $635 \mathrm{~nm}$ illumination. f The corresponding color images from dissected plaque.

accumulation, we performed the MSOT imaging, as previously described in the phantom section. The results in Fig. 4 are shown for three representative specimen, two MMPSense-incubated and one control plaque. Panels $a, b$ and c show cross-sectional MSOT reconstructions, clearly revealing an intra-plaque area with increased signal referred to as 'hot spots' (shown in green), superimposed onto morphological optoacoustic images (made using single 635$\mathrm{nm}$ wavelength). The hot spots are characterized by increased MMP activity as the spectrally resolved MSOT signal originates only from the activated MMPSense ${ }^{\mathrm{TM}} 680$. For validation, epi-fluorescence images of dissected plaque (50- $\mu \mathrm{m}$ sections), superimposed on the corresponding color photographs (panels d, e and f), were done at approximately the same level and match well the corresponding MSOT results. Both MSOT analysis performed on whole activated specimens as well as epi-fluorescence performed on cryosections thus revealed the highest level of MMP activity close to the bifurcation area of the carotid artery. Correspondingly, both MSOT analysis and epi-fluorescence images made on the control specimen did not show fluorescence activity (panels c and f, respectively).

\section{Histopathology}

In order to confirm the MSOT data showing differential MMP activity within an atherosclerotic plaque area, additional histological analyses were performed on the plaque shown in Figs. 3 and 4(b and e). First, in situ zymography was performed on $10-\mu \mathrm{m}$ cryosections to visualize presence of active gelatinases, i.e., MMP's able to degrade gelatin. Based on the MSOT and epi-fluorescence data, two regions of interest were selected based on the presence (denominated as a 'hot-spot') or relative absence (denominated as 'coldspot') of the signal (marked in Fig. 5a with blue and white frames, respectively). Incubation with dye-quenched (DQ)

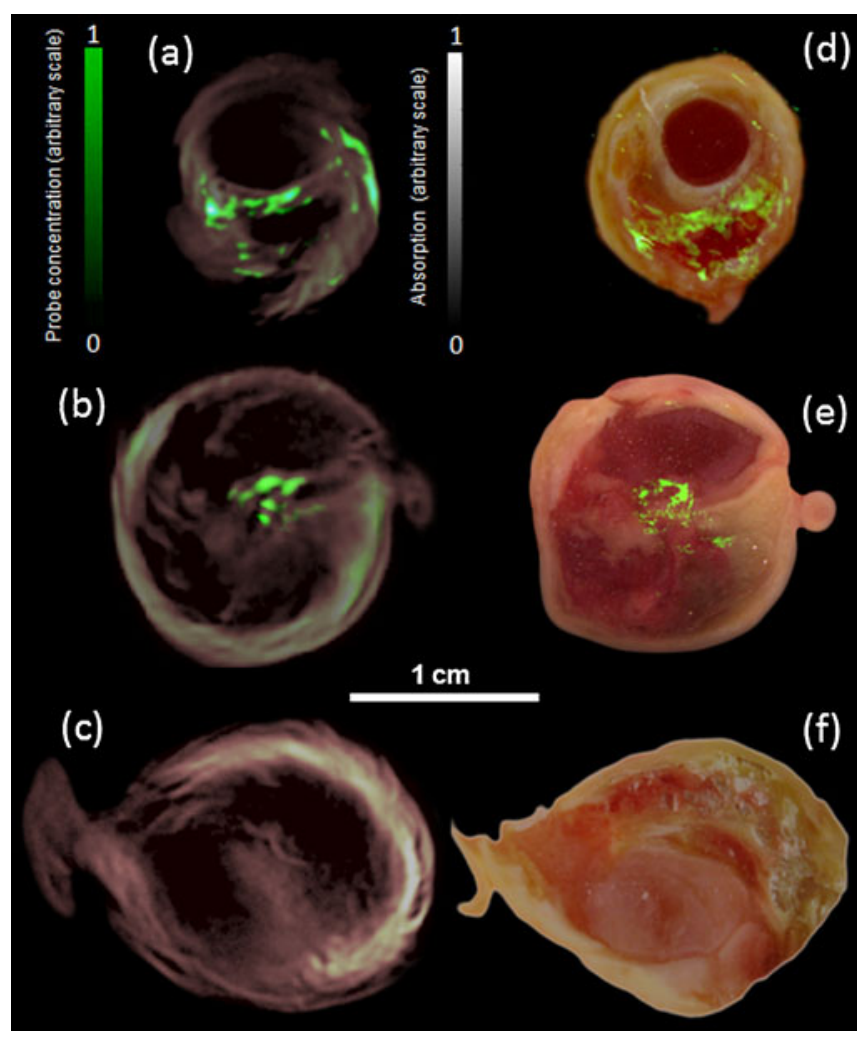

Fig. 4. Localization of MMP activity in three carotid specimen. Samples 1 and 2 were incubated in MMPsense 680 while sample 3 (control) was incubated in PBS. a-c Imaging results from intact plaques made with MSOT. Cross-sectional multispectral reconstruction, revealing location of MMPSense 680 activity in the slice, is shown in green color that is superimposed onto morphological optoacoustic images (made using single 635- $\mathrm{nm}$ wavelength). As expected, samples 1 and 2 show fluorescence from the activated MMPSense, while the control sample has no fluorescence signal. $\mathbf{d}-\mathbf{f}$ The corresponding epi-fluorescent images from dissected plaque (in green) superimposed onto color images of cryosections from the three carotid plaque specimen. The images were done approximately at the same level as in MSOT sessions in (a)-(c). 


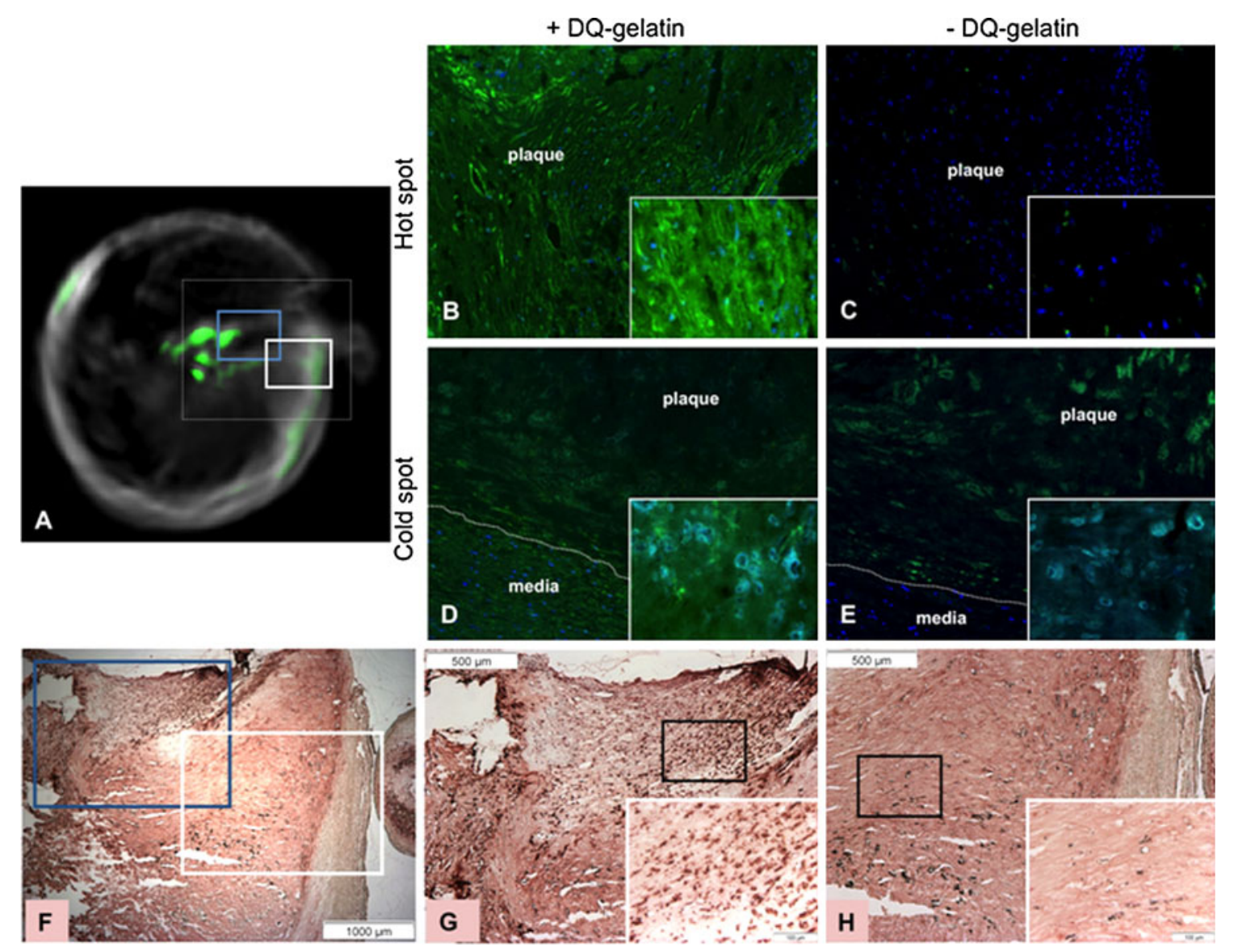

Fig. 5. 'Hot spot' area identified by MSOT (blue frame in a) is characterized by increased gelatinase activity and CD68 ${ }^{+}$ macrophage influx. In situ zymography and immunohistochemistry for CD68 were performed on 10- $\mu$ m cryosections corresponding to the area that revealed enhanced MMP activity as identified by MSOT. b, c Images from cryosections incubated with dye-quenched (DQ) gelatin on the 'hot spot' and 'cold spot' areas, respectively. To check for the presence of autofluorescence, cryosections were also incubated in the absence of DQ gelatin and 'hot spot' (d) and 'cold spot' (e) areas were analyzed. Nuclei were stained with DAPI. Original magnifications: B-E: $\times 160$; insets in B-E: $\times 640$. The CD68 expression in the entire region of interest, indicated by the dotted frame in (a), is shown in (f), where both the 'hot spot' area (blue frame) and 'cold spot' area (white frame) were identified. The 'hot spot' area was characterized by massive macrophage infiltration (g) compared with the 'cold spot' area (h). Insets (g, h): high-power magnifications. Original magnifications: B, $\times 20$; C-D, $\times 40$; insets in C-D, $\times 200$.

gelatin as a substrate for MMP's revealed increased gelatinase activity in the 'hot-spot' area (b) compared with the 'cold-spot' area (c). Within the 'hot-spot', active MMPs were primarily extracellularly located. Within the 'cold-spot' area, active MMP's were detected which were, however, primarily located within the vascular media (c). Although some fluorescence was detected within the plaque area of the 'cold-spot', this is most likely the result of autofluorescence since after incubation in the absence of DQ-gelatin fluorescence was still detected (e). The autofluorescence is probably derived from necrotic cell debris and calcified fragments as also suggested by the aberrant nuclear staining pattern with DAPI and absence of nuclei with normal morphology (c, e). In the 'hot-spot' area autofluorescence was virtually absent (d).

In addition to zymography, we subsequently analyzed whether enhanced MMP activity was associated with an increased macrophage influx. Fig. $5 \mathrm{f}-\mathrm{h}$ shows the region of interest containing both the 'hot-spot' and 'cold-spot' areas. The region of interest presented with a heterogeneous macrophage infiltration pattern, most macrophages being concentrated in the 'hot-spot' area (f). Higher power magnifications revealed massive macrophage influx in the 'hot-spot' area (g), whereas the 'cold-spot' area was almost devoid of macrophages (h). These data suggest and confirm observations by others $[1,12]$ that infiltrating macrophages are the main producers of MMP's thereby contributing to plaque vulnerability.

\section{Discussion}

The indication for a CEA is currently largely based on the combination of symptomatology and degree of stenosis $(>70 \%)$, as defined by flow measurements with duplex ultrasound. These parameters, however, do not provide an accurate assessment of plaque vulnerability and therefore 
only a small percentage of patients do actually benefit from the surgical intervention by preventing a major cerebrovascular event, especially in the asymptomatic group [20,21].

Indeed, introduction of fluorescent molecular probes, sensitive to plaque vulnerability, has proven to provide a generally good indication of the existence of inflammatory processes. Nonetheless, volumetric fluorescence imaging of plaque activity was so far limited by high degree of scattering in large tissue volumes [9, 22], which makes it difficult to accurately localize, quantify and characterize the morphology of the plaque and its vulnerability.

In this report, we have shown that MSOT can resolve with high specificity activated proteases, by using the corresponding absorption changes of activatable 'smart' probes. It can further deliver high-resolution images of activated probes within tissue, herein an optically scattering human carotid specimen. This newly detected capability was found useful in characterizing plaque formations in atherosclerotic disease. Optoacoustic images can simultaneously show the underlining plaque morphology for accurate localization of MMP activity in three dimensions. Furthermore, the MSOT method can simultaneously provide maps of both activated and inactive probe distribution, thus, provide information on molecular contrast and probe biodistribution without introduction of additional blood pool agent. Our future studies will aim at imaging several different probes optimized for different targets. This could potentially be done by using two probes with peak absorption at say 750 and $680 \mathrm{~nm}$ and acquiring multiwavelength optoacoustic data in the spectral windows around 680 and $750 \mathrm{~nm}$. This, however, will certainly require multiple (more than two) wavelengths for efficient spectral unmixing of both probes.

The MSOT findings were verified with a panel of confirmatory studies, i.e., epi-fluorescence imaging of cryosections, zymography and immunohistochemistry. The results from the zymography and immunohistochemistry clearly show a correspondence of MSOT signals and the underlying levels of inflammatory activity, macrophage influx and MMP activity. Results from both MSOT investigations and histological sections confirmed that most of the plaque formation activity occurs, as expected, close to the bifurcation area of the carotid artery.

Even though the current study has dealt with proving the basic feasibility of molecular and morphological characterization of plaques by MSOT, we are currently seeking after in vivo implementation of the method. Here, the presence of blood will introduce additional absorption of light, which will also become more spectrally dependent. This, in turn, will necessitate more sophisticated light attenuation correction and spectral processing algorithms, which take into account absorption spectra of oxy- and deoxyhemoglobin. Some of those issues could potentially be addressed using blind unmixing methods [23]. Moreover, even though the current MSOT imaging geometry has been proven successful for whole-body imaging of living mice [24, 25], clinical translation of the suggested methodology might involve a different technical implementation in terms of light delivery and ultrasonic detection arrangement. This is because most regions of interest in the human body might only be accessible from one side and no full tomographic geometry is available.

Overall, optoacoustics is an inherently three-dimensional visualization tool that has already proven to be capable of penetrating several centimeters into large animals and humans $[11,15]$, thus it can potentially image carotid arteries noninvasively. Furthermore, the spatial resolution of the method is not affected by degree of scattering; thus, it can deliver high-quality data from large diffuse tissue volumes, not accessible by other optical imaging techniques. We therefore foresee our MSOT method to be eventually implemented as a noninvasive diagnostic tool for accurate detection and characterization of cardiovascular disease and biomarker activity, e.g., for in vivo staging of carotid plaque formations for future clinical decision-making.

\begin{abstract}
Acknowledgements. This work was supported by the ERC Independent Researcher Starting Grant (D.R.), the German Research Foundation (DFG) Research Grant [grant number RA1848/1] (D.R.), the ERC Senior Investigator Award (V.N.), and the Medizin Technik BMBF award for excellence in medical innovation (V.N.).
\end{abstract}

Conflict of interest. None.

Open Access. This article is distributed under the terms of the Creative Commons Attribution Noncommercial License which permits any noncommercial use, distribution, and reproduction in any medium, provided the original author(s) and source are credited.

\section{References}

1. Libby P (2002) Inflammation in atherosclerosis. Nature 420:868-874

2. Naghavi M, Libby P, Falk E, Casscells SW, Litovsky S, Rumberger J, Badimon JJ, Stefanadis C, Moreno P, Pasterkamp G, Fayad Z, Stone PH, Waxman S, Raggi P, Madjid M, Zarrabi A, Burke A, Yuan C, Fitzgerald PJ, Siscovick DS, de Korte CL, Aikawa M, Juhani Airaksinen KE, Assmann G, Becker CR, Chesebro JH, Farb A, Galis ZS, Jackson C, Jang IK, Koenig W, Lodder RA, March K, Demirovic J, Navab M, Priori SG, Rekhter MD, Bahr R, Grundy SM, Mehran R, Colombo A, Boerwinkle E, Ballantyne C, Insull W Jr, Schwartz RS, Vogel R, Serruys PW, Hansson GK, Faxon DP, Kaul S, Drexler H, Greenland P, Muller JE, Virmani R, Ridker PM, Zipes DP, Shah PK, Willerson JT (2003) From vulnerable plaque to vulnerable patient: A call for new definitions and risk assessment strategies: Part I. Circulation 108:1664-1672

3. Naghavi M, Libby P, Falk E, Casscells SW, Litovsky S, Rumberger J, Badimon JJ, Stefanadis C, Moreno P, Pasterkamp G, Fayad Z, Stone PH, Waxman S, Raggi P, Madjid M, Zarrabi A, Burke A, Yuan C, Fitzgerald PJ, Siscovick DS, de Korte CL, Aikawa M, Airaksinen KE, Assmann G, Becker CR, Chesebro JH, Farb A, Galis ZS, Jackson C, Jang IK, Koenig W, Lodder RA, March K, Demirovic J, Navab M, Priori SG, Rekhter MD, Bahr R, Grundy SM, Mehran R, Colombo A, Boerwinkle E, Ballantyne C, Insull W Jr, Schwartz RS, Vogel R, Serruys PW, Hansson GK, Faxon DP, Kaul S, Drexler H, Greenland P, Muller JE, Virmani R, Ridker PM, Zipes DP, Shah PK, Willerson JT (2003) From vulnerable plaque to vulnerable patient: A call for new definitions and risk assessment strategies: Part II. Circulation 108:1772-1778

4. Hansson GK, Libby P (2006) The immune response in atherosclerosis: a double-edged sword. Nat Rev Immunol 6:508-519 
5. DeMaria AN, Narula J, Mahmud E, Tsimikas S (2006) Imaging vulnerable plaque by ultrasound. J Am Coll Cardiol 47:C32-C39

6. Cyrus T, Gropler RJ, Woodard PK (2009) Coronary CT angiography (CCTA) and advances in ct plaque imaging. J Nucl Cardiol 16:466-473

7. Botnar RM, Nagel E (2008) Structural and functional imaging by MRI. Basic Res Cardiol 103:152-160

8. Raffel OC, Merchant FM, Tearney GJ, Chia S, Gauthier DD, Pomerantsev E, Mizuno K, Bouma BE, Jang IK (2008) In vivo association between positive coronary artery remodelling and coronary plaque characteristics assessed by intravascular optical coherence tomography. Eur Heart J 29:1721-1728

9. Wallis de Vries BM, Hillebrands JL, van Dam GM, Tio RA, de Jong JS, Slart RH, Zeebregts CJ (2009) Images in cardiovascular medicine. Multispectral near-infrared fluorescence molecular imaging of matrix metalloproteinases in a human carotid plaque using a matrix-degrading metalloproteinase-sensitive activatable fluorescent probe. Circulation 119:e534-e536

10. Levi J, Kothapalli SR, Ma TJ, Hartman K, Khuri-Yakub BT, Gambhir SS (2010) Design, synthesis, and imaging of an activatable photoacoustic probe. J Am Chem Soc 132:11264-11269

11. Razansky D, Vinegoni C, Ntziachristos V (2007) Multispectral photoacoustic imaging of fluorochromes in small animals. Opt Lett 32:2891-2893

12. Jaffer FA, Libby P, Weissleder R (2009) Optical and multimodality molecular imaging: Insights into atherosclerosis. Arterioscler Thromb Vasc Biol 29:1017-1024

13. Razansky D, Distel M, Vinegoni C, Ma R, Perrimon N, Koster R, Ntziachristos V (2009) Multispectral opto-acoustic tomography of deepseated fluorescent proteins in vivo. Nat Photon 3:412-417

14. Wang LV (2009) Photoacoustic imaging and spectroscopy. CRC Press, Boca Raton, Florida
15. Ntziachristos V, Razansky D (2010) Molecular imaging by means of multispectral optoacoustic tomography (MSOT). Chem Rev 110:2783-2794

16. The Laser Institute of America (2000) American national standards for the safe use of lasers. ANSI Z136.1

17. Razansky D, Vinegoni C, Ntziachristos V (2009) Imaging of mesoscopic-scale organisms using selective-plane optoacoustic tomography. Phys Med Biol 54:2769-2777

18. Choudhary S, Higgins CL, Chen IY, Reardon M, Lawrie G, Vick GW 3rd, Karmonik C, Via DP, Morrisett JD (2006) Quantitation and localization of matrix metalloproteinases and their inhibitors in human carotid endarterectomy tissues. Arterioscler Thromb Vasc Biol 26:2351-2358

19. Ntziachristos V, Ripoll J, Wang LV, Weissleder R (2005) Looking and listening to light: The evolution of whole-body photonic imaging. Nat Biotechnol 23:313-320

20. Cina CS, Clase CM, Haynes RB (2000) Carotid endarterectomy for symptomatic carotid stenosis. Cochrane Database Syst Rev CD001081

21. Chambers BR, You RX, Donnan GA (2000) Carotid endarterectomy for asymptomatic carotid stenosis. Cochrane Database Syst Rev CD001923

22. Razansky RN, Rosenthal A, Mallas G, Razansky D, Jaffer FA, Ntziachristos V (2010) Near-infrared fluorescence catheter system for twodimensional intravascular imaging in vivo. Opt Express 18:11372-11381

23. Glatz J, Deliolanis N, Buehler A, Razansky D, Ntziachristos V (2011) Blind source unmixing in multi-spectral optoacoustic tomography. Opt Express 19:3175-3184

24. Ma R, Taruttis A, Ntziachristos V, Razansky D (2009) Multispectral optoacoustic tomography (MSOT) scanner for whole-body small animal imaging. Opt Express 17:21414-21426

25. Razansky D, Buehler A, Ntziachristos V (2011) Volumetric real-time multispectral optoacoustic tomography of biomarkers. Nat Protocols (in press) 